\title{
SARCOPENIA, FRAILTY, AND GERO-SCIENCE: A DECADE OF PROGRESS AND A BRIGHT FUTURE OF DISCOVERY
}

\author{
R.A. FIELDING \\ Corresponding author: Roger A. Fielding, Jean Mayer USDA Human Nutrition Research Center on Aging at Tufts University, Lead Scientist, Nutrition, Exercise Physiology, \\ and Sarcopenia Laboratory, Boston, USA, roger.fielding@tufts.edu
}

J Frailty Aging 2021;10(2)82-83

Published online July 7, 2020, http:/ / dx.doi.org/10.14283/jfa.2020.37

As we reflect 11th edition of the International Conference on Frailty and Sarcopenia Research (ICFSR) in Toulouse (France) $(1,2)$, it is worth reflecting on the progress on sarcopenia and frailty since our inaugural conference in Toulouse in 2011. At that conference (with less than 100 attendees), we were just beginning to understand the observational evidence linking poor muscle function with advancing age to distal outcomes of reduced physical functioning and mobility disability, the underlying biological mechanisms leading to the frailty syndrome were emerging, and pharmacologic and nonpharmacologic intervention trials in patients with sarcopenia and frailty were being planned and initiated. In the nearly one decade since that first meeting, much scientific progress has been made in our understanding of the biological mechanisms of sarcopenia and frailty and a number of therapeutic interventions in this area have been evaluated.

However, it seems like a lifetime ago already since we convened in Toulouse in early March of 2020. The conference had already been adapted to be partially online and in the ensuing days many more of our friends and colleagues informed us that they understandably could no longer make the journey to Toulouse. Little did I know at that time that I would have to depart from France hastily on March 12, remain in quarantine at home for 14 days in the US, and quickly adjust to the new reality that my laboratory was shutting down and ceasing all research activity for an undetermined period of time. My situation pales in comparison to the absolute heroic efforts of my clinical colleagues who are on the frontlines of the COVID-19 pandemic where every day they are putting their lives at risk for the care of their patients.

Fast forward to the 11 th edition of the ICFSR returning to Toulouse in 2020 and it is apparent the fields of frailty and sarcopenia have matured and blossomed. Much work has been done to refine an evidence-based definition of sarcopenia and we are nearing international consensus on these criteria. Furthermore, the syndrome of frailty is well integrated into healthcare systems and clinical practice. Large randomized trials have evaluated lifestyle approaches to treat sarcopenia and frailty with definitive evidence of a benefit on the prevention of major mobility disability. The findings from pharmacologic trials in this space has been more mixed with continued refinement of appropriate pharmacodynamics effects, patient selection, and clinically meaningful outcomes. Progress continues in all these areas with the identification of additional molecular targets that warrant further investigation regarding their function promoting effects. It is heartening to reflect on the spirit of discovery that pervaded the conference despite the fact that many of the presentation where recorded video presentations. Even in the most difficult times the spirit of scientific discovery persists and reassures us that our work seeks to better the lives of people. Perhaps it is important to consider that our work in frailty, resilience, and sarcopenia may help identify the underlying biological phenotype that places individuals at increased risk from the COVID-19 virus.

Superimposed on these accomplishments in frailty and sarcopenia has been the development of a fundamental understanding of the biological pillars of cellular aging and the emergence of gero-science as a thriving area of investigation. As we look to the future, we must work towards integrating the biological targets of senescence and gero-science with our goals of understanding and treating deficits in muscle function, mobility, sarcopenia, and frailty. The integration of these approaches will unify our thinking about age-related syndromes and lead to new discovery and further identification of function promoting therapies to prevent and treat sarcopenia and frailty.

With the continued interest in the development of therapeutic targets aimed at restoring mobility and improving physical functioning in older adults, the forum for scientific discussion provided by the ICFSR is essential. We remain in very early days in the identification of appropriate therapeutic targets in skeletal muscle and in agreement for standardized outcomes to evaluate efficacy in this area. However, consistency is emerging regarding participant selection and the uniformity of outcomes chosen. Indeed, clinical trials of function promoting therapies in older adults continue to mature and the future of discovery is indeed very bright. We can only hope that the scientific process and new discovery will help guide the way for all of mankind in what are clearly very troubled times.

Acknowledgments: Dr. Fielding is supported by the US Department of Agriculture (agreement No. 58-1950-4-003). Any opinions, findings, conclusions, or recommendations expressed in this publication are those of the authors and do not necessarily reflect the view of the USDA.

Conflicts of interest: Dr. Fielding reports grants from National Institutes of Health (National Institute on Aging), during the conduct of the study; grants, personal fees and other from Axcella Health, stock options from Inside Tracker, grants and personal fees from Biophytis, grants and personal fees from Astellas, personal 
fees from Cytokinetics, personal fees from Amazentis, grants and personal fees from Nestle', personal fees from Glaxo Smith Kline, outside the submitted work.

\section{References}

1. Symposia. J Frailty Aging 2020;9:1-45. doi: 10.14283/jfa.2020.8

2. Posters. J Frailty Aging 2020;9:46-179. doi: 10.14283/jfa.2020.9 